\title{
V10 Cost comparison: from pilot to large scale manufacturing of the Brazilian Meningococcal $\mathrm{C}$ conjugate vaccine
}

Renata Chagas Bastos ${ }^{1}$, Milton Neto da Silva ${ }^{1}$, laralice Medeiros de Souza1, Deyves Mendes Paraguassu1, Marcio Roberto Begami Escarlate $^{1}$, José Godinho da Silva Junior ${ }^{1}$, Ricardo de Andrade

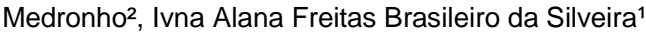

${ }^{1}$ Bio-Manguinhos, Fiocruz, RJ

2 Universidade Federal do Rio de Janeiro - UFRJ;

Introduction: A Brazilian Meningococcal C Conjugate Vaccine has been developed by Bio-Manguinhos based on the epidemiological situation in Brazil. Before starting the Phase III trials, vaccine production was scaled up to obtain reproducible industrial batches.

Objective: This work is related to comparative cost analysis between pilot and industrial scales of steps used to produce Brazilian meningococcal $\mathrm{C}$ conjugate vaccine under GMP.

Methodology: The costs to produce meningococcal $\mathrm{C}$ conjugate bulks (MPCT) in pilot and industrial scales were compared from two perspectives: purification process optimization by tangential flow filtration (TFF) and scaling up reaction volumes in order to obtain intermediate products as oxidized meningococcal $\mathrm{C}$ polysaccharide (MPCO) and hydrazide-activated monomeric tetanus toxin (MATT) as well as the final MPCT. Data related to personnel and raw material costs from fifteen batches of MPCO, nine batches of MATT and four batches of MPCT obtained in pilot scale were compared with the same parameters regarding to five batches of MPCO, nine batches of MATT and three batches of MPCT obtained in large scale.

Results: The five-fold increase in MPCO and MPCT reaction scale resulted in $89 \%$ and $79 \%$ costs reduction, respectively, while 2.5 -fold increase in MATT reaction volume led to a reduction of $43 \%$ in the production costs, based on the relationship between productivity and staff costs. Considering MPCO and MPCT purification steps optimization by TFF, the cost analysis showed a spending reduction of $63 \%$ and $59 \%$, respectively, whereas MATT purification step on the industrial scale led to an increase of $8 \%$ in costs. When taken together personnel and input costs, MPCO production in industrial scale resulted in $69 \%$ cost decrease while MPCT obtained in the same scale was $60 \%$ less expensive. Using the same approach MATT scale up production resulted in an increase of $4 \%$ in costs.

Conclusion: Scaling up of MPCO, MATT and MPCT reaction volume led to a decrease in production costs, as well as MPCO and MPCT purification step optimization by TFF. On the other hand, MATT purification step did not promote a significant reduction in costs, 
suggesting the necessity of improvements in this process. In general, the vaccine production scaling up contributed significantly to cost reduction of downstream process and it would be applied to produce batches in an industrial scale aiming to supply a higher vaccine demand, after phase III clinical trials. This evaluation will allow the global analysis of all investment made in the vaccine development.

Keywords: Cost analysis, Meningococcal Conjugate Vaccine 